\title{
CLASSIFYING A FAMILY OF SYMMETRIC GRAPHS
}

\author{
SANMING ZHOU
}

\begin{abstract}
Let $\Gamma$ be a $G$-symmetric graph admitting a nontrivial $G$-invariant partition $B$ of block size $v$. For blocks $B, C$ of $\mathcal{B}$ adjacent in the quotient graph $\Gamma_{\mathcal{B}}$, let $k$ be the number of vertices in $B$ adjacent to at least one vertex in $C$. In this paper we classify all possibilities for $\left(\Gamma, \Gamma_{\mathcal{B}}, G\right)$ in the case where $k=v-1 \geqslant 2$ and $\mathcal{B}(\alpha)=\mathcal{B}(\beta)$ for adjacent vertices $\alpha, \beta$ of $\Gamma$, where for a vertex of $\Gamma$, say $\gamma \in B, \mathcal{B}(\gamma)$ denotes the set of blocks $C$ such that $\gamma$ is the only vertex in $B$ not adjacent to any vertex in $C$.
\end{abstract}

\section{INTRODUCTION}

A finite graph $\Gamma=(V(\Gamma), E(\Gamma))$ is said to admit a finite group $G$ as a group of automorphisms if $G$ acts on $V(\Gamma)$ in such a way that it preserves the adjacency of $\Gamma$. For such a pair $(\Gamma, G)$, if $G$ is transitive on $V(\Gamma)$ and, in its induced action, is transitive on the set $\operatorname{Arc}(\Gamma)$ of arcs of $\Gamma$, then $\Gamma$ is said to be a $G$-symmetric graph, where an arc is an ordered pair of adjacent vertices. Roughly speaking, in most cases such a graph $\Gamma$ admits a nontrivial $G$-invariant partition, that is, a partition $\mathcal{B}$ of $V(\Gamma)$ such that $1<|B|<|V(\Gamma)|$ and $B^{g} \in \mathcal{B}$ for $B \in \mathcal{B}$ and $g \in G$, where $B^{g}:=\left\{\alpha^{g}: \alpha \in B\right\}$. In this case $\Gamma$ is said to be an imprimitive $G$-symmetric graph. From permutation group theory [3, Corollary 1.5A], this happens precisely when $G_{\alpha}$ is not a maximal subgroup of $G$, where $\alpha \in V(\Gamma)$ and $G_{\alpha}$ is the stabiliser of $\alpha$ in $G$. For such a graph $\Gamma$ we have a natural quotient graph $\Gamma_{\mathcal{B}}$ with respect to $\mathcal{B}$, which is defined to have vertex set $\mathcal{B}$ in which $B, C \in \mathcal{B}$ are adjacent if and only if there exists an edge $\{\alpha, \beta\} \in E(\Gamma)$ with $\alpha \in B$ and $\beta \in C$. In the following we shall always assume that $\Gamma_{\mathcal{B}}$ has at least one edge, so each block of $\mathcal{B}$ is an independent set of $\Gamma$ (see for example [1, Proposition 22.1] and [8]). This quotient graph $\Gamma_{\mathcal{B}}$ conveys a lot of information about the graph $\Gamma$, and in particular it inherits the $G$-symmetry from $\Gamma$ (under the induced action of $G$ on $\mathcal{B}$ ). For $B \in \mathcal{B}$, denote by $\Gamma_{\mathcal{B}}(B)$ the neighbourhood of $B$ in $\Gamma_{\mathcal{B}}$. In introducing a geometric approach to imprimitive symmetric graphs, Gardiner and Praeger [4] suggested an analysis of this quotient graph $\Gamma_{\mathcal{B}}$ together with (i) the 1-design with point set $B$ and "blocks" $\Gamma(C) \cap B$ (with possible repetitions), for all $C \in \Gamma_{\mathcal{B}}(B)$; and (ii) the induced bipartite subgraph $\Gamma[B, C]$ of $\Gamma$ with bipartition $\{\Gamma(C) \cap B, \Gamma(B) \cap C\}$, where $\Gamma(B):=\bigcup_{\alpha \in B} \Gamma(\alpha)$ with $\Gamma(\alpha)$

Received 24th July, 2000

The author appreciates greatly Professor Cheryl E. Praeger for her guidance and excellent supervision.

Copyright Clearance Centre, Inc. Serial-fee code: 0004-9727/01 \$A2.00+0.00. 
the neighbourhood of $\alpha$ in $\Gamma$. Since $\Gamma$ is $G$-symmetric, $\Gamma[B, C]$ is, up to isomorphism, independent of the choice of adjacent blocks $B, C$ of $\mathcal{B}$.

The purpose of this paper is to classify a family of imprimitive symmetric graphs and the corresponding quotients and groups. This makes partial contribution to our project of the study of $G$-symmetric graphs $\Gamma$ with $k=v-1 \geqslant 2$, where $v:=|B|$ is the block size of $\mathcal{B}$ and $k:=|\Gamma(C) \cap B|$ is the size of each part of the bipartition of $\Gamma[B, C]$. It seems that this case is rather rich in both theory and examples: In [7, Section 6] a natural construction of a subclass of such graphs was discovered, and this was further developed in $[9,10]$. In [5] such graphs $\Gamma$ with $\Gamma_{\mathcal{B}}$ a complete graph and $G$ a 3-transitive subgroup of $\operatorname{P\Gamma L}(2, q)$ were determined and characterised, for any prime power $q$. In [11] an intertwined relationship between $G$-symmetric graphs with $k=v-1 \geqslant 2$ and certain kinds of $G$-point- and $G$-block-transitive 1-designs was revealed. For such a graph $\Gamma$ and a vertex $\alpha$ of $\Gamma$, we denote by $B(\alpha)$ the unique block of $\mathcal{B}$ containing $\alpha$. Since $k=v-1 \geqslant 2$, we may define

$$
\mathcal{B}(\alpha):=\{C \in \mathcal{B}: \Gamma(C) \cap B(\alpha)=B(\alpha) \backslash\{\alpha\}\}
$$

and set

$$
m:=|\mathcal{B}(\alpha)| .
$$

Thus $\mathcal{B}(\alpha)$ is the set of blocks of $\mathcal{B}$ which are adjacent to $B(\alpha)$ in $\Gamma_{\mathcal{B}}$ but contain no vertex adjacent to $\alpha$ in $\Gamma$. Since $G$ is transitive on $V(\Gamma)$, the integer $m$ does not depend on the choice of $\alpha$. It seems that the size of $\mathcal{B}(\alpha) \cap \mathcal{B}(\beta)$, for adjacent vertices $\alpha, \beta$ of $\Gamma$, influences a lot the structure of $\Gamma$. For example, we shall see in Lemma 2.1(c) that, if it is greater than $m / 2$, then $\Gamma$ is forced to be an almost cover [9] of $\Gamma_{\mathcal{B}}$, that is, $\Gamma[B, C]$ is a matching of $v-1$ edges. In this paper, we investigate the extreme case where $\mathcal{B}(\alpha)=\mathcal{B}(\beta)$ for adjacent vertices $\alpha, \beta$ of $\Gamma$, and (without loss of generality) $\Gamma_{\mathcal{B}}$ is connected. In this case, we shall prove that the group $G$ is rather restrictive and all of $\Gamma, \Gamma_{\mathcal{B}}$ and $\Gamma[B, C]$ can be determined explicitly, namely $\Gamma \cong(v+1) \cdot K_{m}^{v}, \Gamma_{\mathcal{B}} \cong K_{m}^{v+1}$, $\Gamma$ is an almost cover of $\Gamma_{\mathcal{B}}$, and $G$ is an extension of a group by any 3-transitive group of degree $v+1$ (see Theorem 3.1 and Remark 3.2). Here we denote by $K_{m}^{n}$ the complete $n$-partite graph with $m$ vertices in each part of its $n$-partition, and by $n \cdot \Sigma$ the union of $n$ vertex-disjoint copies of a given graph $\Sigma$.

\section{Preliminary}

For terminology and notation on graphs and permutation groups, the reader is referred to $[\mathbf{1}]$ and $[3]$, respectively. Let $\Gamma$ be a $G$-symmetric graph admitting a nontrivial $G$-invariant partition $\mathcal{B}$ such that $k=v-1 \geqslant 2$. For two vertices $\alpha, \beta$ of $\Gamma$, if $B(\alpha) \in \mathcal{B}(\beta)$ and $B(\beta) \in \mathcal{B}(\alpha)$ hold simultaneously, then we say that $\alpha, \beta$ are mates, and that $\alpha$ is 
the mate of $\beta$ in $B(\alpha)$ (so $\beta$ is the mate of $\alpha$ in $B(\beta)$ as well). Define $\Gamma^{\prime}$ to be the graph with vertex set $V(\Gamma)$ in which $\alpha, \beta$ are adjacent if and only if they are mate. Then $\Gamma^{\prime}$ is $G$-symmetric ([7, Proposition 3]). One can see that the set $\{\mathcal{B}(\alpha): \alpha \in B\}$ is a $G_{B}$-invariant partition of $\Gamma_{B}(B)$, and hence $G_{B}$ induces an action on it, where $G_{B}$ is the setwise stabiliser of $B$ in $G$. Clearly, for $(\alpha, \beta) \in \operatorname{Arc}(\Gamma)$, the value of $|\mathcal{B}(\alpha) \cap \mathcal{B}(\beta)|$ is between 0 and $m$, and is independent of the choice of such $(\alpha, \beta)$ since $\Gamma$ is $G$-symmetric. Part (c) of the following lemma gives an upper bound for this integer in terms of $m$ and the valency of $\Gamma[B, C]$.

LEMma 2.1. Let $(\Gamma, G)$ be as above, and let $s$ be the valency of $\Gamma[B, C]$ (for adjacent blocks $B, C$ of $\mathcal{B}$ ). Then the following (a)-(c) hold.

(a) The valency of $\Gamma$ is equal to $m s(v-1)$, and the valency of $\Gamma_{\mathcal{B}}$ is equal to $m v(\{7$, Theorem 5(a)]).

(b) $G_{B}$ is doubly transitive on $\{\mathcal{B}(\alpha): \alpha \in B\}$ ([7, Theorem 5(b)]).

(c) For $(\alpha, \beta) \in \operatorname{Arc}(\Gamma)$, we have $|\mathcal{B}(\alpha) \cap \mathcal{B}(\beta)| \leqslant m / s$. In particular, if $\mid \mathcal{B}(\alpha) \cap$ $\mathcal{B}(\beta) \mid>m / 2$, then $\Gamma[B, C] \cong(v-1) \cdot K_{2}$.

Proof: We need to prove (c) only. Let $n=|\mathcal{B}(\alpha) \cap \mathcal{B}(\beta)|$ for $(\alpha, \beta) \in \operatorname{Arc}(\Gamma)$. Let $B=B(\alpha), C \in \Gamma_{\mathcal{B}}(B) \backslash \mathcal{B}(\alpha)$, and set $\Gamma(\alpha) \cap C=\left\{\beta_{1}, \ldots, \beta_{s}\right\}$. Then $\mathcal{B}(\alpha) \cap \mathcal{B}\left(\beta_{i}\right)$, for $i=1, \ldots, s$, are pairwise disjoint with each containing $n$ blocks of $\mathcal{B}(\alpha)$. So we have $s n \leqslant m$, as required. In particular, if $n>m / 2$, then we must have $s=1$ and thus $\Gamma[B, C] \cong(v-1) \cdot K_{2}$.

The following example shows that the case where $\mathcal{B}(\alpha)=\mathcal{B}(\beta)$ for adjacent vertices $\alpha, \beta$ of $\Gamma$ can occur. For a finite set $I$, we denote by $I^{(2)}$ the set of ordered pairs of distinct elements of $I$.

EXAMPLE 2.2. Let $X$ be a finite group acting 3-transitively on a finite set $I$ of degree $v+1 \geqslant 4$, and $Y$ a finite group acting on a finite set $J$ of degree $m \geqslant 1$. We require that $Y$ is 2-transitive on $J$ whenever $m \geqslant 2$. Then $G:=X \times Y$ is transitive on $V:=I^{(2)} \times J$ in its action defined by $(i, h, j)^{(x, y)}:=\left(i^{x}, h^{x}, j^{y}\right)$ for $(i, h, j) \in V$ and $(x, y) \in G$. Define $\Gamma$ to be the graph with vertex set $V$ in which $(i, h, j),\left(i^{\prime}, h^{\prime}, j^{\prime}\right)$ are adjacent if and only if $i \neq i^{\prime}$ and $h=h^{\prime}$. Then $\Gamma \cong(v+1) \cdot K_{m}^{v}$, and the assumptions on $X, Y$ imply that $\Gamma$ is $G$-symmetric. Clearly, $\Gamma$ admits $\mathcal{B}:=\{[i, j]: i \in I, j \in J\}$ as a $G$-invariant partition, where $[i, j]:=\{(i, h, j): h \in I \backslash\{i\}\}$. We have $\Gamma_{\mathcal{B}} \cong K_{m}^{v+1}$ with $[i, j],\left[i^{\prime}, j^{\prime}\right]$ adjacent if and only if $i \neq i^{\prime}$. Also, we have $\Gamma[B, C] \cong(v-1) \cdot K_{2}$ for adjacent blocks $B, C$ of $\mathcal{B}$ (hence $k=v-1 \geqslant 2$ ). Moreover, for adjacent vertices $\alpha=(i, h, j), \alpha^{\prime}=\left(i^{\prime}, h, j^{\prime}\right)$ of $\Gamma$, we have $\left.\mathcal{B}(\alpha)=\mathcal{B}\left(\alpha^{\prime}\right)=\{\mid h, \ell]: \ell \in J\right\}$, and hence $|\mathcal{B}(\alpha)|=m$. 


\section{MAIN RESUlt AND THE PROOF}

Unexpectedly, the graphs $\Gamma$ in Example 2.2 are the only $G$-symmetric graphs with $\Gamma_{\mathcal{B}}$ connected such that $k=v-1 \geqslant 2$ and $\mathcal{B}(\alpha)=\mathcal{B}(\beta)$ for adjacent vertices $\alpha, \beta$ of $\Gamma$, and $\Gamma_{\mathcal{B}}, \Gamma[B, C]$ are as shown therein. More precisely, we have the following theorem, which is the main result of this paper.

THEOREM 3.1. Suppose that $\Gamma$ is a $G$-symmetric graph admitting a nontrivial $G$-invariant partition $\mathcal{B}$ such that $k=v-1 \geqslant 2$. Suppose further that $\Gamma_{\mathcal{B}}$ is connected and that $\mathcal{B}(\alpha)=\mathcal{B}(\beta)$ for adjacent vertices $\alpha, \beta$ of $\Gamma$. Let $m=|\mathcal{B}(\alpha)|$. Then $\Gamma \cong(v+1) \cdot K_{m}^{v}$, $\Gamma_{\mathcal{B}} \cong K_{m}^{v+1}, \Gamma[B, C] \cong(v-1) \cdot K_{2}$ for adjacent blocks $B, C$ of $\mathcal{B}$, and the induced action of $G$ on the natural $(v+1)$-partition $\mathbf{B}$ of $\Gamma_{B}$ is 3-transitive. Moreover, the vertices of $\Gamma$ can be labelled by ordered triples of integers such that the following (a)-(c) hold (where we set $I:=\{0,1, \ldots, v\}$ and $J:=\{1,2, \ldots, m\})$ :
(a) $V(\Gamma)=I^{(2)} \times J$, and two vertices $(i, h, j),\left(i^{\prime}, h^{\prime}, j^{\prime}\right) \in V(\Gamma)$ are adjacent in $\Gamma$ if and only if $i \neq i^{\prime}$ and $h=h^{\prime}$.
(b) $\mathcal{B}=\{[i, j]: i \in I, j \in J\}$, where $[i, j]:=\{(i, h, j): h \in I \backslash\{i\}\}$, and $[i, j],\left[i^{\prime}, j^{\prime}\right]$ are adjacent blocks if and only if $i \neq i^{\prime}$.
(c) $\mathbf{B}=\{\mathbf{i}: i \in I\}$, where $\mathbf{i}=\{[i, j]: j \in J\}$.

Conversely, the graph $\Gamma$ defined in (a) together with the group $G=X \times Y$ satisfies all conditions of the theorem, where $X$ is a group acting 3-transitively on $I, Y$ is a group acting on $J$ which is 2-transitive if $m \geqslant 2$, and the action of $G$ on $V(\Gamma)$ is as defined in Example 2.2.

Proof: By our assumption we have $|\mathcal{B}(\alpha) \cap \mathcal{B}(\beta)|=m>m / 2$ for $(\alpha, \beta) \in \operatorname{Arc}(\Gamma)$. Thus Lemma 2.1(c) implies

(i) $\Gamma[D, E] \cong(v-1) \cdot K_{2}$ for adjacent blocks $D, E$ of $\mathcal{B}$.

Let $B$ be a block of $\mathcal{B}$ and let $\alpha_{1}, \alpha_{2}, \ldots, \alpha_{v}$ be vertices of $B$. For each $\alpha_{i} \in B$, we label (in an arbitrary way) the $m$ blocks in $\mathcal{B}\left(\alpha_{i}\right)$ by $[i, j], j \in J$. Also, we label the unique mate $\beta_{i j}$ of $\alpha_{i}$ in the block $[i, j]$ by $(i, 0, j), j \in J$. For each block $[i, j]$ and for each $h \in I \backslash\{0\}$ distinct from $i$, (i) implies that $[i, j]$ contains a unique vertex adjacent to $\alpha_{h}$. We label such a vertex in $[i, j]$ by $(i, h, j)$. In view of (i) one can see that each vertex in $[i, j]$ receives a unique label, and that the labels of distinct vertices in $[i, j]$ have distinct second coordinates. Therefore, for each $i \in I \backslash\{0\}$ and $j \in J$, we may identify the block $[i, j]$ with the set $\{(i, h, j): h \in I \backslash\{i\}\}$. By our assumption, for $i, h \in I \backslash\{0\}$ with $i \neq h$ and $j \in J$, we have

(ii) $\mathcal{B}((i, h, j))=\mathcal{B}\left(\alpha_{h}\right)=\{[h, 1],[h, 2], \ldots,[h, m]\}$.

In particular, this implies that

(iii) $[i, j],\left[i^{\prime}, j^{\prime}\right]$ are adjacent blocks, for distinct $i, i^{\prime} \in I \backslash\{0\}$ and any $j, j^{\prime} \in J$. Moreover, if two vertices $(i, h, j),\left(i^{\prime}, h^{\prime}, j^{\prime}\right)$ are adjacent, then by (ii) and our assumption we must have $\mathcal{B}\left(\alpha_{h}\right)=\mathcal{B}((i, h, j))=\mathcal{B}\left(\left(i^{\prime}, h^{\prime}, j^{\prime}\right)\right)=\mathcal{B}\left(\alpha_{h^{\prime}}\right)$, which is true only when 
$h=h^{\prime}$. This, together with (i) and (iii), implies the following assertion.

(iv) For distinct $i, i^{\prime} \in I \backslash\{0\}$ and any $j, j^{\prime} \in J$, two labelled vertices $(i, h, j),\left(i^{\prime}, h^{\prime}, j^{\prime}\right)$ of $\Gamma$ are adjacent if and only if $h=h^{\prime}$. In other words, for adjacent blocks $D=[i, j], E=\left[i^{\prime}, j^{\prime}\right]$ of $\mathcal{B}$, the bipartite subgraph $\Gamma[D, E]$ of $\Gamma$ is the matching of $v-1$ edges joining $(i, h, j)$ and $\left(i^{\prime}, h, j^{\prime}\right)$, for $h \in I \backslash\left\{i, i^{\prime}\right\}$.

Therefore, $\left(i, i^{\prime}, j\right)$ and $\left(i^{\prime}, i, j^{\prime}\right)$ are mates and hence, for the graph $\Gamma^{\prime}$ defined at the beginning of the previous section, we have

(v) $\Gamma^{\prime}((i, h, j))=\left\{\left(h, i, j^{\prime}\right): j^{\prime} \in J\right\}$.

Now let us examine a particular labelled vertex, say $(i, h, j)$. From Lemma 2.1(a) and (i) above, the valency of $\Gamma$ is $m(v-1)$, and hence the neighbourhood $\Gamma((i, h, j))$ of $(i, h, j)$ contains $m(v-1)$ vertices. From (iv) we have $\left\{\left(i^{\prime}, h, j^{\prime}\right): i^{\prime} \in I \backslash\{0, h, i\}\right.$, $\left.j^{\prime} \in J\right\} \subseteq \Gamma((i, h, j))$ and this contributes $m(v-2)$ neighbours of $(i, h, j)$. Note that $\alpha_{h}$ is also a neighbour of $(i, h, j)$. Apart from these, there are $m-1$ remaining neighbours of $(i, h, j)$, which we denote by $\delta_{2}, \ldots, \delta_{m}$, respectively. By (i) these vertices $\delta_{2}, \ldots, \delta_{m}$ belong to distinct blocks, say $B_{2}, \ldots, B_{m}$, of $\mathcal{B}$. For each $\delta_{t}$, we have $\mathcal{B}\left(\delta_{t}\right)=\mathcal{B}((i, h, j))=$ $\mathcal{B}\left(\alpha_{h}\right)=\{[h, 1],[h, 2], \ldots,[h, m]\}$ by (ii) and our assumption. In particular, this implies that all the blocks $[h, \ell]$, for $\ell \in J$, are adjacent to the block $B_{t}$. On the other hand, from (v) we have $\Gamma^{\prime}\left(\left(h, h^{\prime}, \ell\right)\right)=\left\{\left(h^{\prime}, h, t\right): t \in J\right\}$ for each vertex $\left(h, h^{\prime}, \ell\right) \in[h, \ell] \backslash\left\{\beta_{h \ell}\right\}$. In other words, the $m$ mates of each vertex in $[h, \ell] \backslash\left\{\beta_{h \ell}\right\}$ are in $\bigcup_{h^{\prime} \in I \backslash\{0, h\}, \ell \in J}\left[h^{\prime}, t\right]$. So the only possibility is that $\beta_{h \ell}$ is the mate of $\delta_{t}$ in $[h, \ell]$, for each $\ell \in J$. Consequently, we have

(vi) $\mathcal{B}\left(\beta_{h 1}\right)=\cdots=\mathcal{B}\left(\beta_{h m}\right)=\left\{B, B_{2}, \ldots, B_{m}\right\}$, and hence none of $B, B_{2}, \ldots, B_{m}$ coincides with $[i, j]$ for any $i \in I \backslash\{0\}$ and $j \in J$.

We know from (iii) that the blocks $\left[i^{\prime}, j^{\prime}\right]$, for $i^{\prime} \in I \backslash\{0, h\}$ and $j^{\prime} \in J$, are all adjacent to $[h, \ell]$. Besides these $m(v-1)$ blocks, $B, B_{2}, \ldots, B_{m}$ are the only blocks of $\mathcal{B}$ adjacent to $\{h, \ell]$ in $\Gamma_{\mathcal{B}}$ since $\Gamma_{\mathcal{B}}$ has valency $m v$ (Lemma 2.1(a)). Therefore, if we apply the procedure above to another vertex $\left(i^{\prime}, h, j^{\prime}\right)$, we would get the same blocks $B_{2}, \ldots, B_{m}$. In other words, these blocks are independent of the choice of the vertex $(i, h, j)$ (depending only on $h$ ), and hence they are adjacent to the block $[i, j]$ for any $i \in I \backslash\{0\}$ and $j \in J$. Moreover, since the mate $\delta_{t}$ of $\beta_{h \ell}$ in $B_{t}$ is unique, the vertices $\delta_{2}, \ldots, \delta_{m}$ are also independent of the choice of $(i, h, j)$ and thus they are common neighbours of all such vertices $(i, h, j)$. Thus, since the valency of $\Gamma_{\mathcal{B}}$ is $m v, B, B_{2}, \ldots, B_{m}$ are the only unlabelled blocks of $\mathcal{B}$. From this and by a similar argument to that above, we see that for each $h \in I \backslash\{0\}$, all the vertices $(i, h, j), i \in I \backslash\{0, h\}, j \in J$, have a common neighbour in each $B_{l}$, which we now label by $(0, h, t)$. Since for distinct $h, h^{\prime}$ the vertices $(i, h, j),\left(i, h^{\prime}, j\right)$ have different neighbours in $B_{t}$, the vertices of $B_{t}$ receive pairwise distinct labels. Now let us label $B, B_{2}, \ldots, B_{m}$ with $[0,1],[0,2], \ldots,[0, m]$, respectively, and label each $\alpha_{h}$ with $(0, h, 1)$. 
Then all the vertices of $\Gamma$ and all the blocks of $\mathcal{B}$ have been labelled. From the labelling above, the validity of (a) and (b) follows immediately.

Since the valency of $\Gamma$ is $m(v-1)$, the argument above also shows that for each $h \in I$ the connected component of $\Gamma$ containing the vertex $\alpha_{h}$ is the complete $v$-partite graph $K_{m}^{v}$ with $v$-partition $\{\{(i, h, j): j \in J\}: i \in I\}$, where we set $\alpha_{0}=\beta_{11}$. Hence we have $\Gamma \cong(v+1) \cdot K_{m}^{v}$. Also, $\Gamma_{\mathcal{B}}$ is the complete $(v+1)$-partite graph $K_{m}^{v+1}$ with $(v+1)$-partition $\mathbf{B}:=\{\mathbf{i}: i \in I\}$, where $\mathbf{i}:=\mathcal{B}\left(\alpha_{i}\right)=\{[i, j]: j \in J\}$ for $i \in I$. Clearly, $\left(\Gamma_{B}\right)_{\mathbf{B}} \cong K_{v+1}$ and $\mathbf{B}$ is a $G$-invariant partition of $\mathcal{B}$. From Lemma 2.1(b), $G_{B}$ is doubly transitive on $\{\mathcal{B}(\gamma): \gamma \in B\}$. The setwise stabiliser in $G$ of the block 0 contains $G_{B}$ as a subgroup, and so is doubly transitive on the neighbourhood $\mathbf{B} \backslash\{0\}$ of 0 in $\left(\Gamma_{\mathcal{B}}\right)_{\mathbf{B}}$. Therefore, $G$ is 3-transitive on $\mathbf{B}$.

Finally, for $G=X \times Y$ with $X$ triply transitive on $I$ and $Y$ doubly transitive on $J$ whenever $m \geqslant 2$, Example 2.2 shows that the graph $\Gamma$ defined in (a) satisfies all the conditions in the theorem.

REMARK 3.2. In Theorem 3.1, $G$ may or may not be faithful on $\mathbf{B}$. (This can be seen from Example 2.2, where the action of $G$ on B is permutationally isomorphic to the action of $X$ on $I$ which is not necessarily faithful.) Let $K$ be the kernel of the action of $G$ on $\mathrm{B}$, and set $H:=G / K$. Then $H$ is 3-transitive and faithful on $\mathbf{B}$ of degree $v+1$, and $G$ is an extension of $K$ by $H$. From the classification of finite highly transitive permutation groups (see for example $[\mathbf{2}, \mathbf{6}]), H$ is one of the following: $S_{v+1}(v \geqslant 3), A_{v+1}(v \geqslant 4)$, $\mathrm{M}_{v+1}(v=10,11,21,22,23), \mathrm{M}_{11}(v=11), \operatorname{AGL}(d, 2)\left(v=2^{d}-1\right), \mathbb{Z}_{2}^{4} \cdot A_{7}(v=15)$, and $\operatorname{PSL}(2, v) \leqslant H \leqslant \operatorname{P} \Gamma(2, v)$ ( $v$ a prime power). Example 2.2 shows that $m=|\mathcal{B}(\alpha)|$ defined in (2) can be any positive integer and $H$ can be any group listed above.

\section{REFERENCES}

[1] N.L. Biggs, Algebraic graph theory, (Second edition) (Cambridge University Press, Cambridge, 1993).

[2] P.J. Cameron, 'Finite permutation groups and finite simple groups', Bull. London Math. Soc. 13 (1981), 1-22.

[3] J.D. Dixon and B. Mortimer, Permutation groups (Springer-Verlag, Berlin, Heidelberg, New York, 1996).

[4] A. Gardiner and C.E. Praeger, 'A geometrical approach to imprimitive graphs', Proc. London Math. Soc. (3) 71 (1995), 524-546.

[5] A. Gardiner, C.E. Praeger and S. Zhou, 'Cross ratio graphs', Proc. London Math. Soc. (to appear).

[6] W.M. Kantor, 'Homogeneous designs and geometric lattices', J. Combin. Theory Ser. A 38 (1985), 66-74.

[7] C.H. Li, C.E. Praeger and S. Zhou, 'A class of finite symmetric graphs with 2-arc transitive quotients', Math. Proc. Cambridge Philos. Soc. 129 (2000), 19-34.

[8] C.E. Praeger, 'Imprimitive symmetric graphs', Ars Combin. 19A (1985), 149-163. 
[9] S. Zhou, 'Almost covers of 2-arc transitive graphs', (submitted).

[10] S. Zhou, 'Imprimitive symmetric graphs, 3-arc graphs and 1-designs', Discrete Math. (to appear).

[11] S. Zhou, 'Constructing a class of symmetric graphs', (submitted).

Department of Mathematics and Statistics

The University of Western Australia

Perth, WA 6907

Australia

e-mail: smzhou@maths.uwa.edu.au
Current address:

Department of Mathematics and Statistics

The University of Melbourne

Parkville, VIC 3010

Australia

e-mail: smzhou@ms.unimelb.edu.au 